\title{
Quantum efficiency enhancement in CsI/metal photocathodes
}

\author{
Lingmei Kong, Alan G. Joly, Timothy C. Droubay, Wayne P. Hess* \\ Physical Sciences Division, Pacific Northwest National Laboratory, \\ P.O. Box 999, Richland, Washington 99352, USA
}

\begin{abstract}
:
High quantum efficiency enhancement is found for hybrid metal-insulator photocathodes consisting of thin films of CsI deposited on $\mathrm{Cu}(100), \mathrm{Ag}(100), \mathrm{Au}(111)$ and $\mathrm{Au}$ films irradiated by $266 \mathrm{~nm}$ laser pulses. Low work functions (near or below $2 \mathrm{eV}$ ) are observed following ultraviolet laser activation. Work functions are reduced by roughly $3 \mathrm{eV}$ from that of clean metal surfaces. We discuss various mechanisms of quantum efficiency enhancement for alkali halide/metal photocathode systems and conclude that the large change in work function, due to Cs accumulation of Cs metal at the metal-alkali halide interface, is the dominant mechanism for quantum efficiency enhancement.
\end{abstract}

*Email: wayne.hess@pnnl.gov

\section{Introduction}

In order to meet requirements for next-generation free electron laser (FEL) and synchrotron-based light sources, and to enable dynamic transmission electron microscopy 
(DTEM) $[1,2]$, robust new photocathode materials are needed. Current photocathode materials with high quantum efficiency (QE), such as Cs:GaAs cathodes, are highly sensitive to low concentrations of background gases, and thus require extreme vacuum conditions $\sim 10^{-11}$ Torr for long-term operation [1]. Even under extreme vacuum conditions, the lifetime of currently used photocathodes is limited.

Metal photocathodes have excellent tolerance to common background gases, are robust toward pulsed laser excitation and display long operational lifetimes. However, bare metal photocathodes have relatively high work functions $(>4 \mathrm{eV})$ and low $\mathrm{QE}$, typically on the order of $10^{-5}$ to $10^{-4}[1,3]$, which results in the requirement of a UV drive laser with high average power and hence limited application. In order to overcome limitations of metal photocathodes, several approaches have been explored. These include incorporation of nanostructured features, such as gratings and nanohole arrays, to couple light efficiently into the metal and induce surface-plasmon enhanced electric fields [4,5], utilizing ultrathin oxide layers to reduce work function [2], and deposition of alkali halide films to increase QE [6-10]. Alkali halide thin film deposition has been proven to be one of the most effective ways to lower work function and increase QE of metal photocathodes.

Quantum efficiencies of metal single crystals (for example, $\mathrm{Cu}, \mathrm{Cr}$ and $\mathrm{Mo}$ ) can be greatly enhanced by the deposition of alkali halide films, such as $\mathrm{CsBr}$ [6-8], followed by UV irradiation. Three mechanisms have been proposed for the observed QE enhancement in $\mathrm{CsBr}$ covered metals: (i) a decrease in work function of the hybrid-metal alkali halide system compared to the bare metal. Work function is reduced by deposition of the alkali 
halide film itself and is further reduced following laser irradiation. (ii) direct photoemission from the intraband states (F centers) created during laser irradiation might contribute to part of the QE enhancement. (iii) formation of a thin Cs layer at the metalalkali halide interface or at the hybrid photocathode surface. Thin Cs layers are commonly used to enhance photoemission from a variety of photocathode materials.

Recently, much higher QE enhancement and significantly greater work function reduction has been found for a $\mathrm{CsI}$ thin film, compared to a $\mathrm{CsBr}$ film, on $\mathrm{Cu}$ single crystal substrates [9]. Direct photoemission from the $\mathrm{Cu} 3 d$ band, for the CsI thin film, and electrons emitted from the intraband states were considered as possible contributions although several key mechanistic questions remain. In order to determine the dominant QE enhancement mechanisms, we explore the deposition of CsI thin film on noble metal substrates $(\mathrm{Cu}(100), \mathrm{Ag}(100), \mathrm{Au}(111)$ and a $\mathrm{Au}$ film on $\mathrm{Si})$. We find that the major mechanism for $\mathrm{QE}$ enhancement is a dramatic work function reduction, due to accumulation of $\mathrm{Cs}$ metal at the metal/CsI interface. For $\mathrm{Cu}$ and $\mathrm{Au}$ substrates, much of the d-band electron density is available for photoemission, using $4.66 \mathrm{eV}$ photoexcitation, as work functions are reduced to near $2 \mathrm{eV}$. We find that $\mathrm{F}$ centers (intraband states) do not contribute significantly to photoemission enhancement of $\mathrm{CsI} /$ metal photocathode systems.

\section{Experimental details}

All single crystal metal substrates were repeatedly Ar ion sputtered and annealed $\left(560^{\circ} \mathrm{C}\right.$ for $\mathrm{Cu}, 200^{\circ} \mathrm{C}$ for $\mathrm{Ag}$ and $\left.\mathrm{Au}\right)$ in ultrahigh vacuum until $\mathrm{X}$-ray photoelectron spectroscopy data displayed only substrate core-levels. Gold films of $100 \mathrm{~nm}$ thickness 
were deposited on clean Si substrates by argon plasma sputtering. CsI thin films were then grown on the clean substrates by heating $99.999 \%$ pure CsI powder in an effusion cell to $410^{\circ} \mathrm{C}$ in a separate growth chamber. A quartz crystal microbalance positioned near the growth location was used to calibrate the film growth rate. No reaction of CsI with any of the metal substrates is observed. The coated sample was then irradiated by an unfocused $266 \mathrm{~nm}$ pulsed laser (pulse width 400 picoseconds, repetition rate of $20 \mathrm{kHz}$, beam diameter of $1.5 \mathrm{~mm}$ ) for several hours, to induce laser activation. Laser activation (LA) required between 5 and 10 hours in vacuum, at a laser power of $12 \mathrm{~mW}$, depending upon metal substrate type for nominal $8 \mathrm{~nm}$ thick films. Base pressure increased only slightly to $2 \times 10^{-9}$ Torr during LA.

Relative photoelectron yields and kinetic-energy distributions were obtained using a photoelectron spectrometer with hemispherical analyzer (Kratos Axis HS) and the same $266 \mathrm{~nm}$ laser as used in the activation process but with a greatly reduced average power $(20 \mu W)$. Work functions were determined from the photoemission spectra by subtracting the width of the energy-resolved photoemission peak, at the base, from the photon energy $(4.66 \mathrm{eV})$ and correcting for the instrument resolution. X-ray photoelectron spectroscopy (Al K $\alpha$ X-rays) was used to confirm film thicknesses and characterize iodine and cesium concentrations during the laser activation process. The $\mathrm{QE}$ of laser activated $\mathrm{CsI} / \mathrm{Cu}$ was determined by measuring the photoelectron current using an electrometer. Quantum efficiency of laser activated $\mathrm{CsI}$ thin films on $\mathrm{Cu}$ was determined, as a function of $\mathrm{CsI}$ film thickness (ranging between 6 and $40 \mathrm{~nm}$ ).

\section{Results and discussion}


Figure 1 displays the QE for $8 \mathrm{~nm}$ CsI films on four different substrates: $\mathrm{Cu}(100)$, $\mathrm{Ag}(100), \mathrm{Au}(111)$ and a $\mathrm{Au}$ film on $\mathrm{Si}$ as a function of laser activation time. Significant QE enhancement is induced by LA for CsI on all the four different substrates. QE after LA varies on the different substrates, especially for $\mathrm{CsI}$ on $\mathrm{Cu}(100)$, which results in the highest photoelectron yield. QE after LA for CsI on the other three substrates are nearly the same. No significant difference was observed between single crystal $\mathrm{Au}(111)$ and the polycrystalline gold metal film substrate ( $\mathrm{Au}$ film on $\mathrm{Si}$ ). The time needed for full LA varied with each metal substrate requiring approximately 3 hours for CsI on $\operatorname{Ag}(100), 7$ hours for $\mathrm{CsI}$ on $\mathrm{Cu}$ and 10 hours for CsI on $\mathrm{Au}$.

Figure 2 shows the photoemission kinetic energy spectra for bare metal substrates and laser activated CsI on the four different substrates as a function of LA time. These photoemission spectra regardless of substrate show similar features. Each spectrum broadens significantly as LA proceeds, for all four CsI/metal systems, which indicates a reduction in the work function resulting from LA. In addition, for all substrates, a dominant low kinetic energy feature develops as LA proceeds. This feature dominates the kinetic energy spectra and photoelectron yield in fully laser activated CsI/metal systems.

Table I displays initial and final work functions and quantum efficiencies for CsI on $\mathrm{Cu}(100), \mathrm{Ag}(100), \mathrm{Au}(111)$ and a $\mathrm{Au}$ film on Si. A significant work function reduction and QE enhancement was observed for CsI deposited on all the substrates. CsI on $\mathrm{Cu}(100)$ results in the lowest final work function $(\sim 1.7 \mathrm{eV})$ and highest final $\mathrm{QE}$ $\left(7.5 \times 10^{-3}\right)$. Slightly greater work functions of $1.9,2.0$ and $2.1 \mathrm{eV}$ are observed for 
$\mathrm{CsI} / \mathrm{Au}$ film/Si, CsI/Au(111), and CsI/Ag(100) with QEs of $3.1 \times 10^{-3}, 2.7 \times 10^{-3}$ and 2.8 x $10^{-3}$, respectively.

The results of hybrid CsI/metal photocathodes strongly contrast those of $\mathrm{CsBr} /$ metal [6-9] as CsI/metal photocathodes have significantly higher quantum efficiencies following laser activation. Figure 3 presents the energy diagram of the $\mathrm{CsI} / \mathrm{Cu}$ system. As a result of the large work function reduction induced by $\mathrm{LA}$, electrons located in the $\mathrm{Cu}$ $d$-band can be directly emitted following excitation by $4.66 \mathrm{eV}$ photons. Direct photoemission from the electron dense $d$-band greatly enhances the photoemission QE at $4.66 \mathrm{eV}$ for laser activated $\mathrm{CsI} / \mathrm{Cu}$. However, for $\mathrm{CsBr} /$ metal systems the work function is not reduced sufficiently, even after prolonged laser activation, to enable direct electron emission from the $\mathrm{Cu} d$-band.

Table II displays work function reduction, relative photoelectron yield and laser activation (LA) time for $\mathrm{CsI}$ on $\mathrm{Au}(111)$ with different film thicknesses $(6,10,20$ and 40 $\mathrm{nm})$. The term relative photoelectron yield refers to the photoelectron yield after LA with respect to that of a $6 \mathrm{~nm}$ CsI film on $\mathrm{Au}(111)$. Photoelectron yield decreases monotonically with film thickness, and drops $90 \%$ as film thickness varies from 6 to 40 $\mathrm{nm}$, whereas final work function values after LA increase from $1.85 \mathrm{eV}$ to $2.2 \mathrm{eV}$. The LA time needed to achieve full activation increases with film thickness.

Figure 4 displays a time sequence of the photoelectron spectra for $8 \mathrm{~nm}$ CsI on four different substrates: $\mathrm{Cu}(100), \operatorname{Ag}(100), \mathrm{Au}(111)$ and the polycrystalline $\mathrm{Au}$ film on $\mathrm{Si}$. Photoelectron spectra both before and after laser activation are displayed. In addition, spectra obtained 10 hours after laser activation is completed, and then "reactivated" for 
one $(\mathrm{CsI} / \mathrm{Ag})$ or three hours $(\mathrm{CsI} / \mathrm{Cu}(100), \mathrm{CsI} / \mathrm{Au}(111)$ and $\mathrm{CsI} / \mathrm{Au}$ film $)$ is presented in Figure 4. The data show a clear decrease in photoelectron yield 10 hours after laser irradiation is completed. This indicates that the conditions necessary for large quantum yield enhancement are transient at room temperature, over the course of hours, even under ultrahigh vacuum conditions. In all cases, the enhanced QE could be nearly fully recovered by additional laser activation.

We note that the relative loss of $\mathrm{QE}$ enhancement, over 10 hours following the conclusion of laser activation, is substrate dependent. The greatest loss is observed for $\mathrm{CsI} / \mathrm{Cu}(100)$ followed by $\mathrm{CsI} / \mathrm{Ag}(100)$ and $\mathrm{CsI} / \mathrm{Au}(111)$ and finally the $\mathrm{CsI} / \mathrm{Au}$ thin film on $\mathrm{Si}$. Furthermore, the rate of loss, immediately following conclusion of laser activation, is also substrate dependent, with faster loss rates observed for $\mathrm{CsI} / \mathrm{Ag}(100)$ than for $\mathrm{CsI} / \mathrm{Au}$ systems. It is clear that the differences in relative $\mathrm{QE}$ loss and rate of $\mathrm{QE}$ loss must be due to chemical and/or physical differences at the CsI/metal interface. These results would not vary between the four systems if Cs metal accumulated on the CsI surface.

We can draw several conclusions from analysis of the above data. The large reduction in work function is highly correlated with $\mathrm{QE}$ enhancement. If the work function of the hybrid CsI/metal photocathode is sufficiently large, following LA, electrons can be directly emitted from the substrate metal $d$ band and easily escape the CsI overlayer into the vacuum (mean free path for a $<2 \mathrm{eV}$ electron is roughly $20 \mathrm{~nm}$ in CsI). Photoemission from the electron dense d-band is likely an important contribution to the $\mathrm{QE}$ enhancement for $\mathrm{CsI}$ on $\mathrm{Cu}$ and $\mathrm{Au}$ as previously suggested [9]. When the work 
function is decreased to near $2 \mathrm{eV}, \mathrm{Cu} 3 d$ and $\mathrm{Au} 5 \mathrm{~d}$ electrons can be directly emitted following excitation by $4.66 \mathrm{eV}$ photons. This mechanism results in the appearance of the intense low kinetic energy features displayed in Figure 2, as both $\mathrm{Cu} 3 d$ and $\mathrm{Au} 5 d$ band are located $\sim 2.0 \mathrm{eV}$ below the Fermi level, $\mathrm{E}_{\mathrm{F}}[14]$. We note that if the metal $d$ band is the only electron source for the large QE enhancement, then the QE enhancement behavior for CsI on $\mathrm{Ag}$ should be different because the $\mathrm{Ag} 4 d$ band is located $4.0 \mathrm{eV}$, rather than $2.0 \mathrm{eV}$, below $\mathrm{E}_{\mathrm{F}}$, [14] implying that strong d-band emission from $\mathrm{Ag}$ is less likely. While photoemission from $\operatorname{CsI} / \operatorname{Ag}(100)$ is significantly reduced from that of $\mathrm{CsI} / \mathrm{Cu}(100)$, under similar conditions, it is nearly as intense as from $\mathrm{CsI} / \mathrm{Au}$ systems in contrast to this prediction. Nonetheless we expect photoemission from CsI/Ag(100) to increase sharply with the large reduction in work function, that accompanies LA as is observed.

The large work function reduction for CsI on metal substrates, induced by LA, is most likely caused by Cs metal accumulation into an ultrathin layer [9]. Regarding Cs accumulation during LA, there are two hypotheses. Hypothesis 1: Cesium accumulates on the top of the CsI layer. If this is true and the CsI valence band contributes strongly, a thicker layer should have higher quantum yield. As noted previously, this is not observed (see Table II). Hypothesis 2: Cesium accumulates at the interface between the metal substrate and the cesium iodide. If this is true, then the thicker layer should reduce the quantum efficiency due to reduced electron transport through the thicker film. Hypothesis 2 is consistent with all data reported in Table II. Very low coverage of Cs on metal substrates (less than 0.5 monolayer) is known to reduce the metal work function to as low as $1.6 \mathrm{eV}$ [15-17]. 
In total, the results, including work function decreases, thickness studies, relative loss of $\mathrm{QE}$ enhancement and the rate of $\mathrm{QE}$ loss at the conclusion of laser activation, argue strongly for a model where Cs metal accumulates at the interface between the metal and alkali halide film (Hypothesis 2). For example, the QE enhancement of laser activated $\mathrm{CsI} / \mathrm{Cu}(100)$ decreases significantly over the course of several hours in vacuum. If Cs atoms do accumulate at the interface, then accumulated Cs atoms might migrate into the CsI film lattice or diffuse away from the irradiated area following laser activation. We note that the rate of $\mathrm{QE}$ loss and the time required for asymptotic laser activation (Figure 2) varies significantly with noble metal substrate. Moreover, the activation time varies with the substrate. CsI on $\mathrm{Ag}$ activates fastest, followed by CsI on $\mathrm{Cu}$ and $\mathrm{CsI}$ on $\mathrm{Au}$. These observed differences likely result from Cs accumulation chemistry due to specific interactions between each metal substrate and the generated Cs layer. Furthermore, alkali metal atoms accumulated on the alkali halide surface (the interface between alkali halide and vacuum) are known to desorb readily at room temperature to maintain alkali halide stoichiometry under UV irradiation [18-20]. This fact, while not confirmed specifically for $\mathrm{Cs}$ metal on $\mathrm{CsBr}$ or $\mathrm{CsI}$, strongly suggests that the alkali halide remains stoichiometric at the alkali halide/vacuum interface and that Cs metal accumulates at the metal/alkali halide interface.

Intraband states (e.g. F center defects) created by laser activation could possibly contribute to the QE enhancement in alkali halide/metal systems $[6,8,10]$. F center states are located at $3.37 \mathrm{eV}$ and $1.9 \mathrm{eV}$ below the bottom of conduction band of $\mathrm{CsBr}[10,11]$ and CsI $[12,13]$ respectively (below the respective Fermi levels by roughly 1.0 and 1.3 $\mathrm{eV}$ ). Since both $\mathrm{F}$ bands are located below the respective metal Fermi levels, we expect $\mathrm{F}$ 
center states to be "full" for activated alkali halide/metal systems. In other words, anion vacancies, created by photoemission from F center states, should be refilled by electrons from the metal Fermi level. Thus, in principle, photoemission from F centers, could be efficient provided certain conditions are met. Namely, there must be a sufficient number of $\mathrm{F}$ center states created by laser activation, their optical cross section must be high, and electron refilling of the anion vacancy must be rapid.

It does not appear, from our experimental data, that these conditions are met. There are few $\mathrm{F}$ centers created in the thin film volume as the maximum density is very likely below $1 \%$ of total anion sites. A very low total number of $\mathrm{F}$ center states are therefore expected in the thinnest alkali halide films tested. While the thinnest CsI film studied in this work is $6 \mathrm{~nm}$, we have investigated $3 \mathrm{~nm}$ films previously [9] and obtained similar results, i.e. very high QE enhancement. The $3 \mathrm{~nm}$ films support only 6 or 7 monolayers of CsI such that even fewer F centers likely exist per unit area. Although there should be more total $\mathrm{F}$ centers created in thicker films, the data in Table II show a decrease in photoelectron yield for thicker films, rather than the increase that would be predicted if $\mathrm{F}$ centers contributed significantly. The last argument also applies to any other intraband defect states (e.g. H, H aggregate, M, or I, centers) that could possibly contribute to the QE enhancement mechanism. Therefore, intraband states, created by LA, are not a significant source of electron photoemission at $4.66 \mathrm{eV}$ from hybrid alkali halide/metal photocathodes.

\section{Conclusions}


Cesium metal very likely accumulates at the metal/alkali halide interface during laser activation of hybrid CsI/metal photocathodes. The large work function reduction due to Cs accumulation during laser activation is therefore the dominant factor for the very high QE enhancement in CsI/metal systems. Direct electron emission from the metal $d$-band, made possible by the large work function reduction, is likely responsible for the high QE enhancement of laser activated hybrid CsI/metal photocathodes. F center emission can only contribute very modestly to QE enhancement and, more generally, intraband states created during LA are not a significant source of photoemission.

\section{Acknowledgements:}

This research is part of the Chemical Imaging Initiative conducted under the Laboratory Directed Research and Development Program at Pacific Northwest National Laboratory (PNNL) and was performed using EMSL, a national scientific user facility sponsored by the Department of Energy's Office of Biological and Environmental Research located at PNNL, a multiprogram national laboratory operated by Battelle Memorial Institute for the U.S. Department of Energy.

\section{References:}

[1] D. H. Dowell, I. Bazarov, B. Dunham, K. Harkay, C. Hernandez-Garcia, R. Legg, H. Padmore, T. Rao, J. Smedley, and W. Wan, Nucl. Instrum. Methods Phys. Res. A 622, 685 (2010).

[2] A. Polyakov, C. Senft, K. F. Thompson, J. Feng, S. Cabrini, P. J. Schuck, H. A. Padmore, S. J. Peppernick, and W. P. Hess, Phys. Rev. Lett. 110, 076802 (2013).

[3] J. Le Perchec, P. Quémerais, A. Barbara, and T. López-Ríos, Phys. Rev. Lett. 100, 066408 (2008). 
[4] R. K. Li, H. To, G. Andonian, J. Feng, A. Polyakov, C. M. Scoby, K. Thompson, W. Wan, H. A. Padmore, and P. Musumeci, Phys. Rev. Lett. 110, 074801 (2013).

[5] K. Németh, K. C. Harkay, M. van Veenendaal, L. Spentzouris, M. White, K. Attenkofer, and G. Srajer, Phys. Rev. Lett. 104, 046801 (2010).

[6] J. R. Maldonado, Z. Liu, D. H. Dowell, R. E. Kirby, Y. Sun, P. Pianetta, and F. Pease, Phys. Rev. ST Accel. Beams 11, 060702 (2008).

[7] W. He, S. Vilayurganapathy, A. G. Joly, T. C. Droubay, S. A. Chambers, J. R. Maldonado, and W. P. Hess, Appl. Phys. Lett. 102, 071604 (2013).

[8] J. R. Maldonado, P. Pianetta, D. H. Dowell, J. Smedley, and P. Kneisel, J. Appl. Phys. 107, 013106 (2010).

[9] L. Kong, A. G. Joly, T. C. Droubay, Y. Gong, W. P. Hess Appl. Phys. Lett. 104, 171106 (2014)

[10] J. R. Maldonado, Z. Liu, D. H. Dowell, R. E. Kirby, Y. Sun, P. Pianetta, and F. Pease, Microelectron. Eng. 86, 529 (2009).

[11] Z. Liu, Y. Sun, P. Pianetta, J. R. Maldonado, R. F. W. Pease, and S. Schuetter, Appl. Phys. Lett. 90, 231115 (2007).

[12] H.W. Sarkas, L.H. Kidder, and K. H. Bowen, J. Chem. Phys. 102, 1 (1995)

[13] H. R. Philipp and F. A. Taft, J. Phys. Chem. Solids, 1, 159163 (1956)

[14] F.L. Battye, A. Goldmann, L. Kasper, and S. Hüfner, Z. Physik B 27, 209-214 (1977).

[15] S. Å. Lindgren and L. Walldén, Phys. Rev. B 45, 6345 (1992).

[16] S. Å. Lindgren and L. Walldén, Solid State Commun. 25, 13 (1978).

[17] R. Q. Wu and D. S. Wang, Phys. Rev. B 41, 12541 (1990).

[18] B. Such, P. Czuba, P. Piatkowski, and M. Szymonski, Surf. Sci. 451, 203 (2000).

[19] W.P. Hess, A.G. Joly, K.M. Beck, M. Henyk, P.V. Sushko, and A.L. Shluger, Surf. Sci. 564, 62 (2004).

[20] B. Such, J. Kolodziej, P. Czuba, P. Piatkowski, P. Struski, F. Krok, and M. Szymonski, Phys. Rev. Lett. 85, 2621 (2000). 


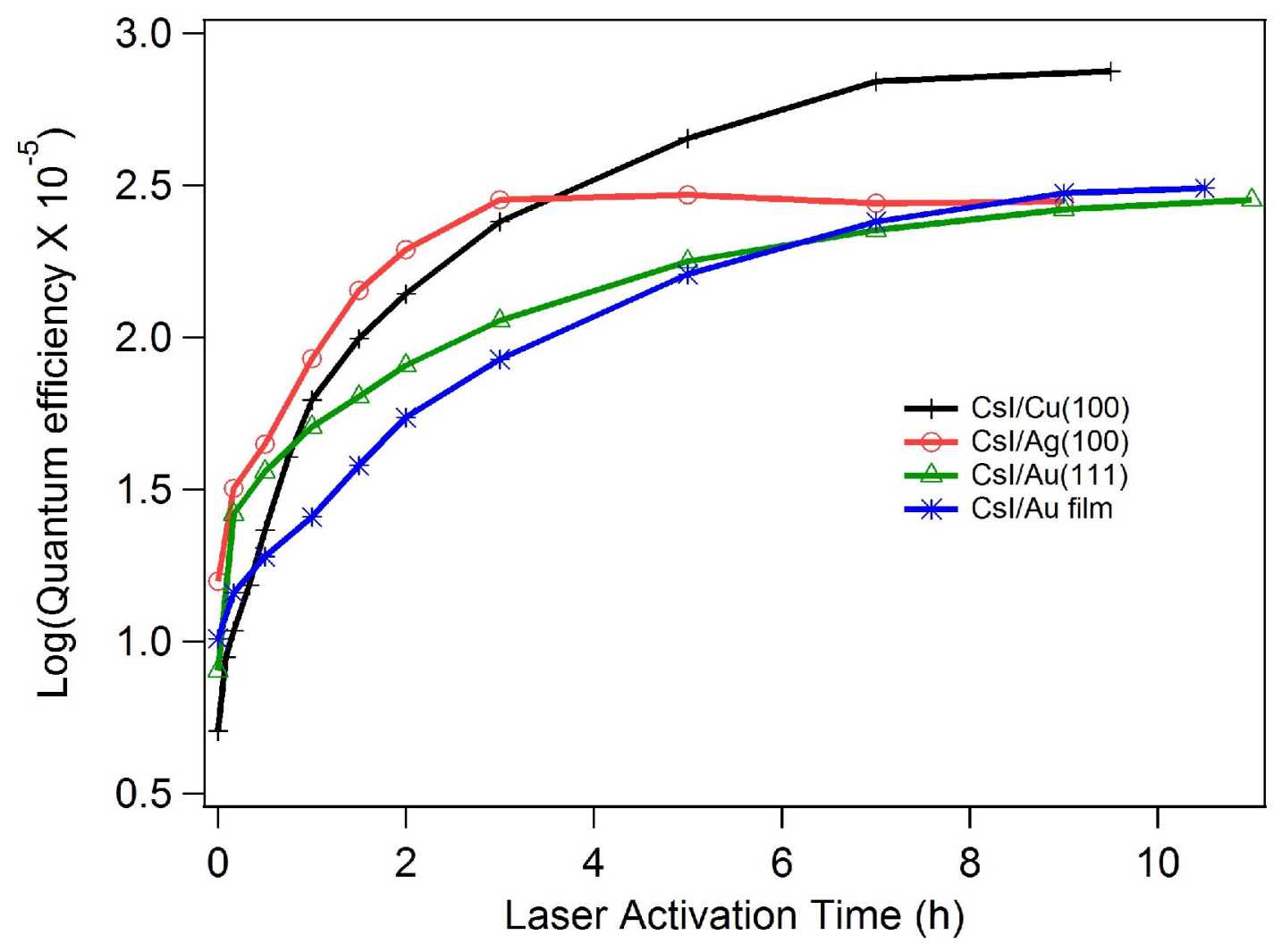

Figure 1. Quantum efficiency of $8 \mathrm{~nm}$ CsI covered $\mathrm{Cu}(100), \mathrm{Ag}(100), \mathrm{Au}(111)$ and $\mathrm{Au}$ film on $\mathrm{Si}$ as a function of laser activation (LA) time. The measurements were taken using a $266 \mathrm{~nm}$ pulsed laser source with average power of $20 \mu \mathrm{W}$ and the activation utilized the same laser with average power of $12 \mathrm{~mW}$. The maximum QE for each sample was determined by photoelectron current measurement and all curves were normalized according to the obtained maximum $\mathrm{QE}$. 
(a)

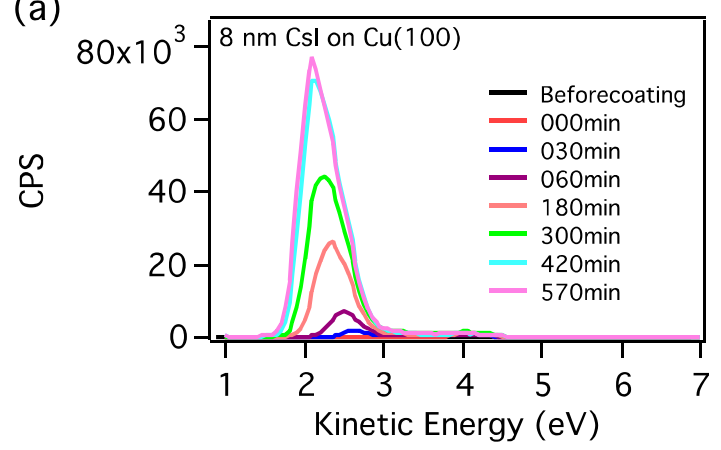

(c)

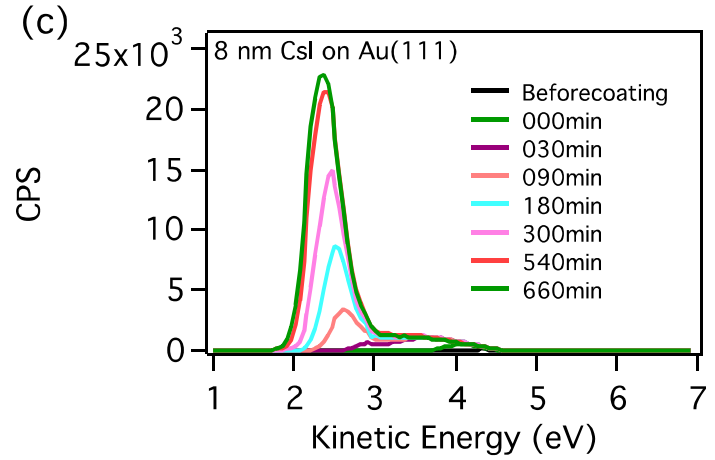

(b)

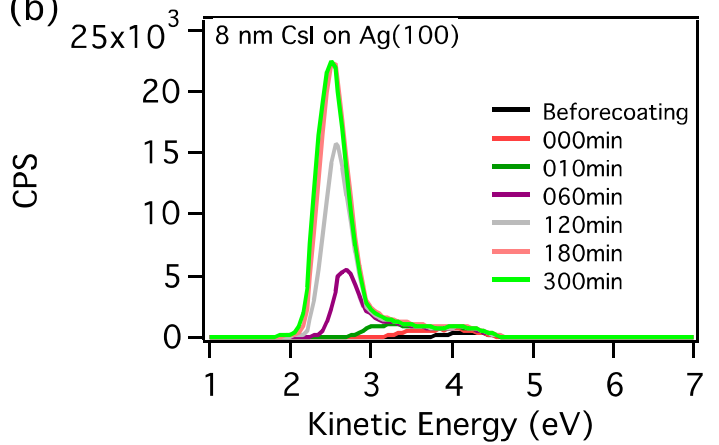

(d)

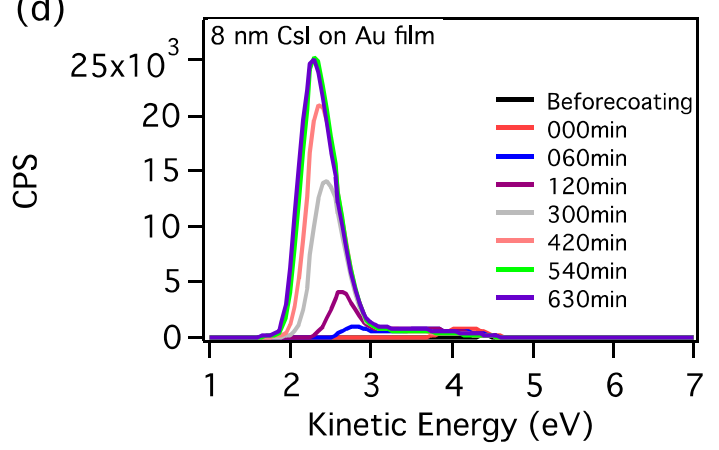

Figure 2. Photoelectron yield (in counts per second, CPS) for $8 \mathrm{~nm} \mathrm{CsI} \mathrm{on} \mathrm{Cu}$ (100), $\mathrm{Ag}(100), \mathrm{Au}(111)$ and $\mathrm{Au}$ film on $\mathrm{Si}$ with different laser activation (LA) times compared to bare substrates. The measurement was made using a $266 \mathrm{~nm}$ pulsed laser source with average power of $20 \mu W$ and the activation utilized the same laser with average power of $12 \mathrm{~mW}$. 


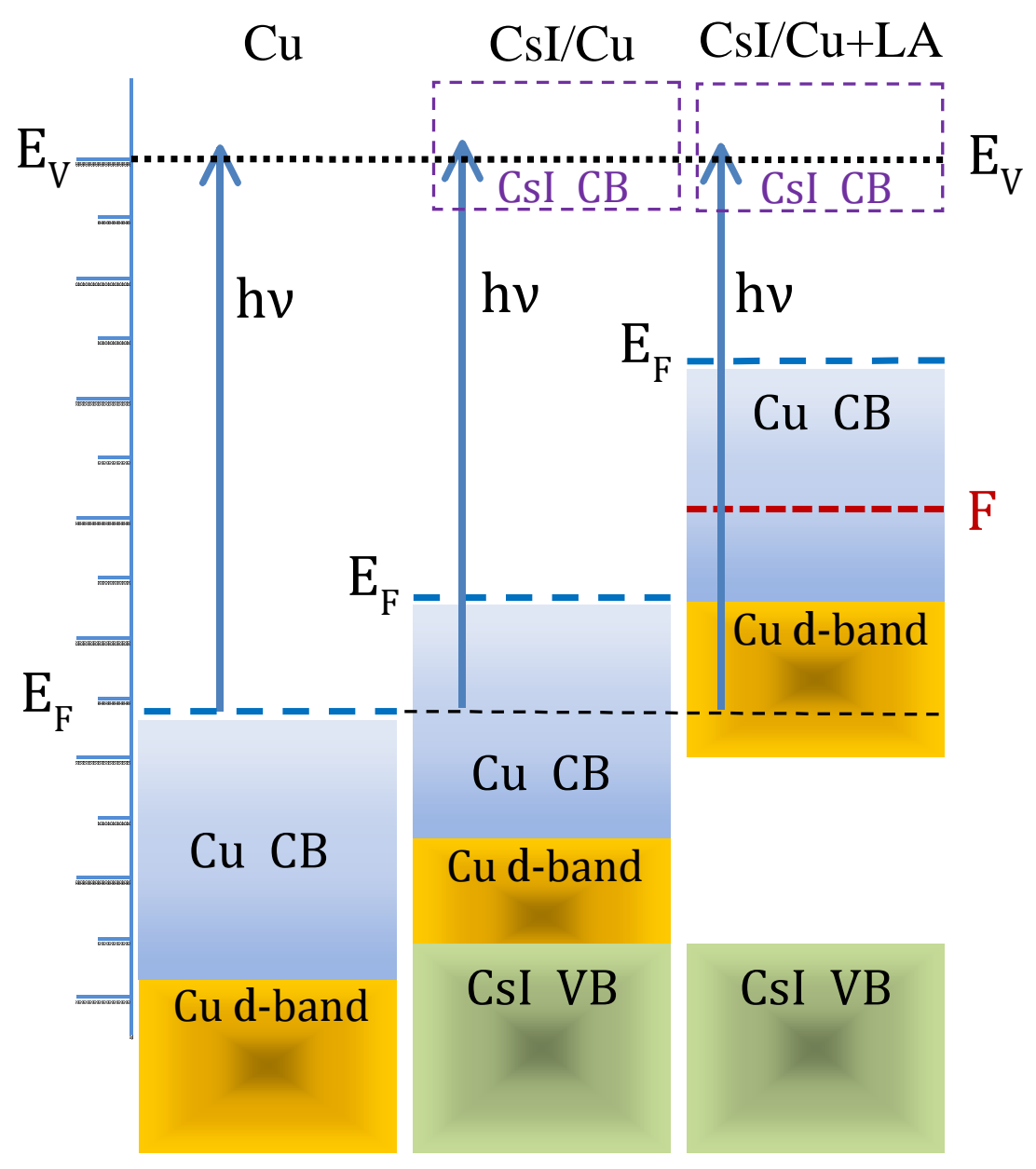

Figure 3. Energy diagrams of clean $\mathrm{Cu}$ metal, a thin film of $\mathrm{CsI}$ on $\mathrm{Cu}$ metal $(\mathrm{CsI} / \mathrm{Cu})$ and a laser activated thin film $\mathrm{CsI}$ on $\mathrm{Cu}$ metal $(\mathrm{CsI} / \mathrm{Cu}+\mathrm{LA}) . \mathrm{E}_{\mathrm{F}}, \mathrm{E}_{\mathrm{V}}$, and $\mathrm{F}$ denote the Fermi level, the vacuum level and the F center level in CsI. The photon energy $(4.66 \mathrm{eV})$ is represented by the arrows and hv. The position and width of the $\mathrm{Cu} d$-band is represented by the gold rectangle while the green and dashed purple rectangles indicate the CsI valence and conduction bands. The Fermi and $d$-band levels rise toward the vacuum level as the work function is reduced, first by addition of a thin film of CsI (middle panel) and then by laser activation of the $(\mathrm{CsI} / \mathrm{Cu})$ system (right panel). A 4.66 $\mathrm{eV}$ photon is capable of inducing photoemission from the $\mathrm{Cu} d$-band for the laser activated $(\mathrm{CsI} / \mathrm{Cu})$ system. 

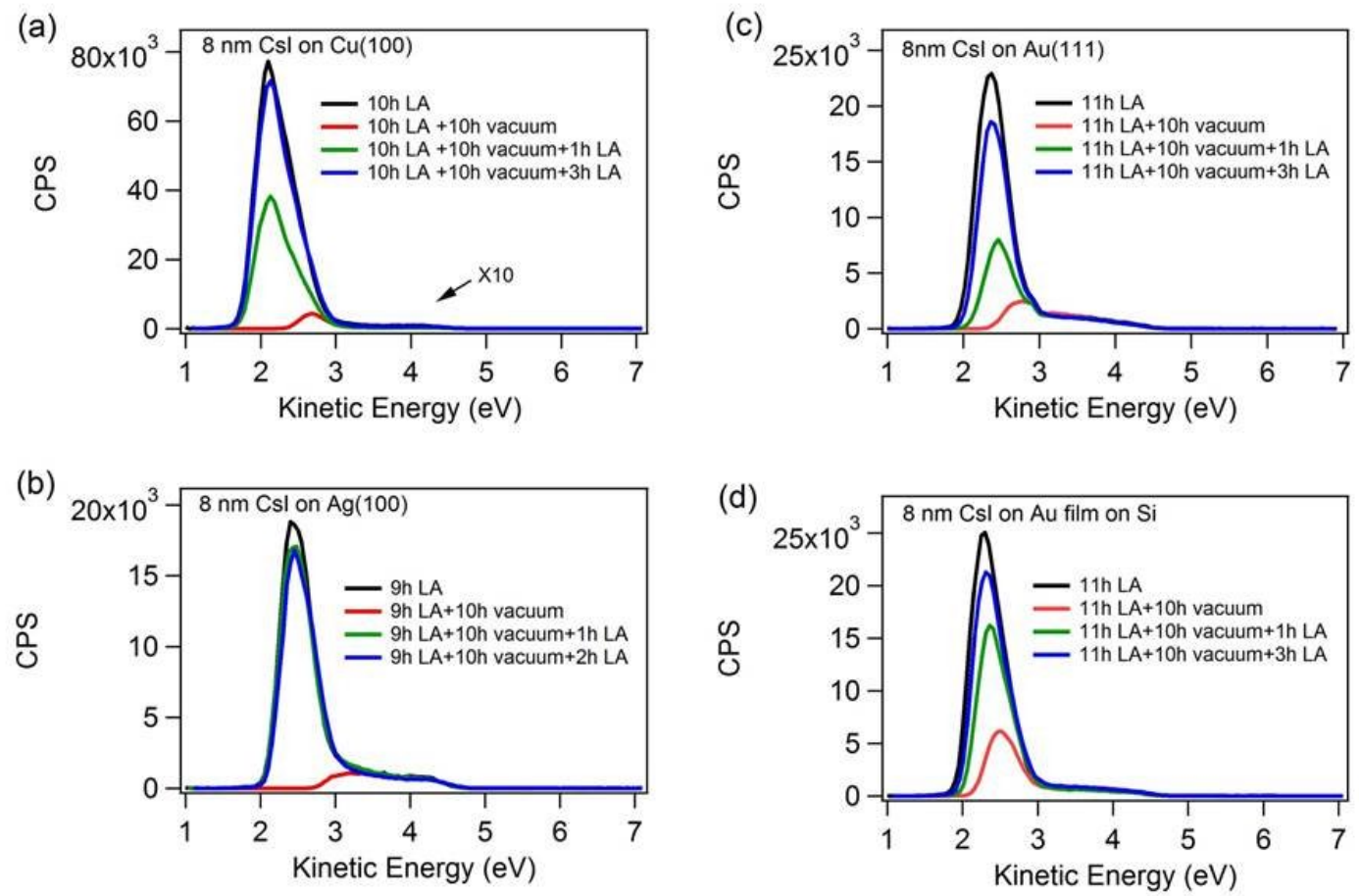

Figure 4. Photoelectron yield (in counts per second, CPS) versus photoelectron kinetic energy for $8 \mathrm{~nm}$ CsI on $\mathrm{Cu}(100), \mathrm{Ag}(100), \mathrm{Au}(111)$ and $\mathrm{Au}$ film on Si. Black spectra: after laser activation; Red spectra: laser activation stopped for 10 hours in vacuum; Green spectra and Blue spectra: additional laser activation for 1 hour and 3 hours, respectively. 
Table I. Work function reduction and quantum efficiency enhancement for $8 \mathrm{~nm}$ CsI on $\mathrm{Cu}(100), \mathrm{Ag}(100), \mathrm{Au}(111)$ and $\mathrm{Au}$ film on Si.

\begin{tabular}{lllll}
\hline & $\begin{array}{l}\text { Initial work } \\
\text { function }(\mathbf{e V})\end{array}$ & $\begin{array}{l}\text { Final work } \\
\text { function }(\mathbf{e V})\end{array}$ & $\begin{array}{l}\text { QE before LA } \\
\left(\times \mathbf{1 0}^{-3}\right)\end{array}$ & $\begin{array}{l}\text { QE after LA } \\
\left(\times \mathbf{1 0}^{-\mathbf{3}}\right)^{*}\end{array}$ \\
\hline $\mathrm{CsI} / \mathrm{Cu}(100)$ & 4.65 & 1.7 & 0.050 & 7.5 \\
$\mathrm{CsI} / \mathrm{Ag}(100)$ & 4.26 & 2.1 & 0.16 & 2.8 \\
$\mathrm{CsI} / \mathrm{Au}(111)$ & 5.1 & 2.0 & 0.079 & 2.7 \\
$\mathrm{CsI} / \mathrm{Au} \mathrm{film}$ & & 1.9 & 0.10 & 3.1 \\
\hline \hline
\end{tabular}

* QE obtained by photoelectron current measurement

Table II. Work function reduction, relative photoelectron yield and laser activation (LA) time as a function of CsI film thickness on $\mathrm{Au}(111)$.

\begin{tabular}{llll}
\hline \hline Thickness (nm) & $\begin{array}{l}\text { Final work } \\
\text { function (eV) }\end{array}$ & $\begin{array}{l}\text { Relative } \\
\text { Photoelectron } \\
\text { yield* }\end{array}$ & LA Time (h) \\
\hline 6 & 1.85 & 1.0 & 11 \\
10 & 1.85 & 0.95 & 14 \\
20 & 2.0 & 0.35 & 18 \\
40 & 2.2 & 0.1 & 24 \\
\hline \hline
\end{tabular}

* Relative photoelectron yield refers to photoelectron yield after LA compared to $6 \mathrm{~nm}$ CsI film covered $\mathrm{Au}(111)$ 
Hybrid Photocathodes of CsI thin films on noble metals show high quantum efficiency Quantum efficiencies of $\sim 1 \%$ are observed for the $\mathrm{CsI} / \mathrm{Cu}(100)$ system $(6 \mathrm{~nm}$ film) Hybrid photocathodes hold promise for light sources and dynamic electron microscopes 


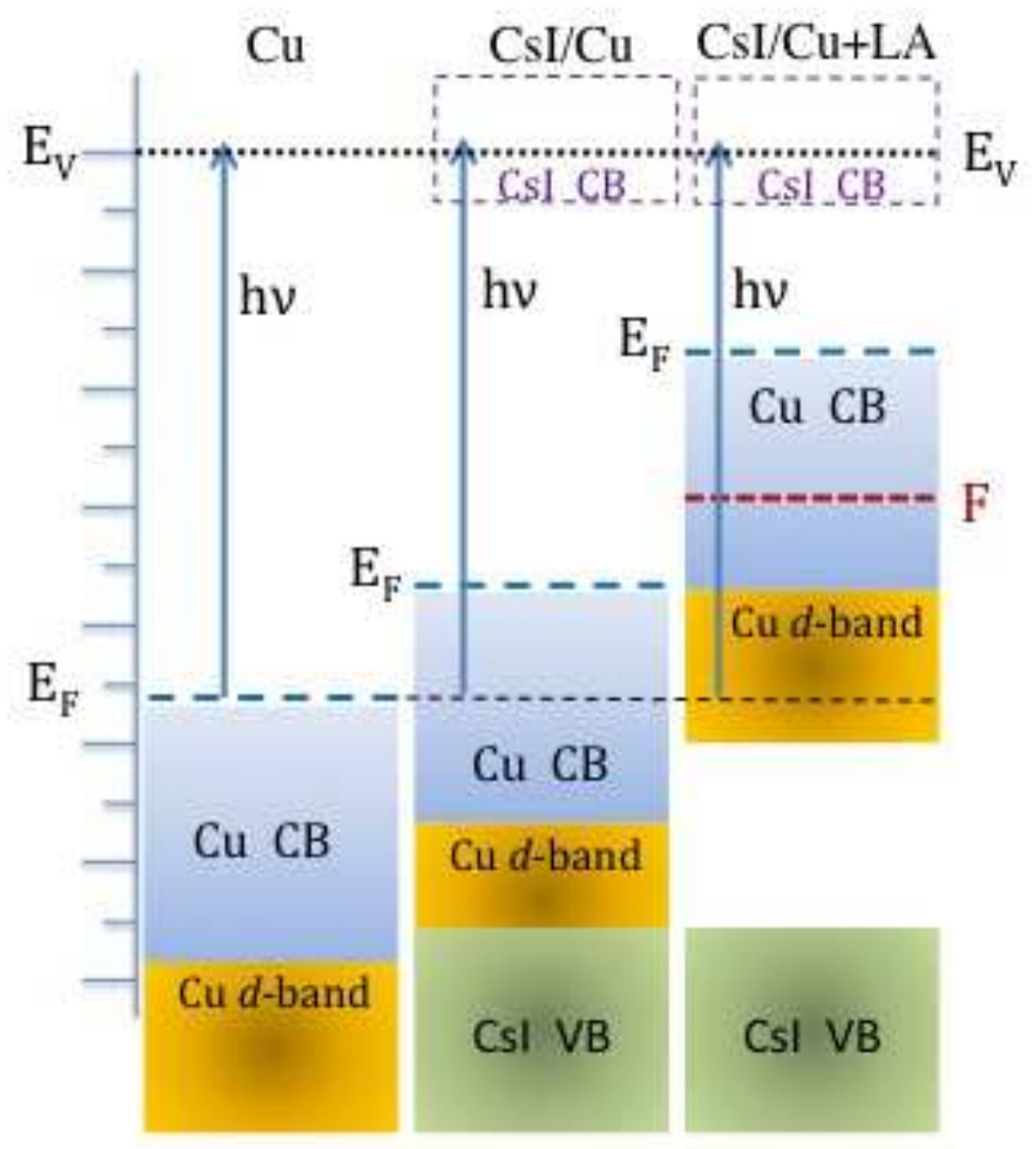

